\title{
Estrategia híbrida en el manejo de cardiopatías congénitas. Resultados iniciales en una experiencia multicéntrica
}

\author{
Hybrid Strategy in the Management of Congenital Heart Defects. Initial Results in a \\ Multicentric Experience
}

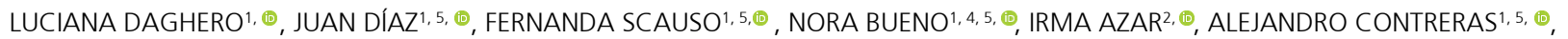

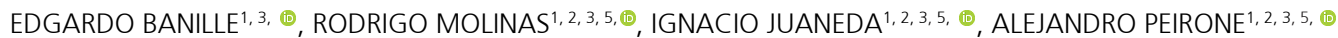

\begin{abstract}
RESUMEN
Introducción: El abordaje híbrido implica la colaboración estrecha de los equipos quirúrgico y de hemodinamia para el tratamiento de cardiopatías congénitas complejas, con el objetivo de disminuir el número de intervenciones a través de técnicas menos invasivas y de evitar las limitaciones dadas por el tamaño de los accesos vasculares y la utilización de circulación extracorpórea. Objetivos: Evaluar indicaciones, técnicas, resultados iniciales y seguimiento a corto plazo del abordaje híbrido.

Material y métodos: Revisión retrospectiva de pacientes abordados de manera híbrida desde marzo de 2014 hasta septiembre de 2020. Se realizaron 14 procedimientos en 13 pacientes. Edad media de 16,8 meses (1 día-13 años), el 50\% fueron neonatos y el 57\% de sexo masculino; el peso medio fue $9 \mathrm{~kg}(1,9-70 \mathrm{~kg})$. Los procedimientos híbridos realizados incluyeron implante de stent en coartación de aorta (4 pacientes), implante de stent ductal (3 pacientes), implante de stent ramas pulmonares (1 paciente), valvuloplastia aórtica neonatal (4 pacientes) y cierre de comunicación interventricular muscular ( 2 pacientes). El acceso se realizó mediante disección carotídea en 8 procedimientos y esternotomía en los 6 restantes.

Resultados: Todos los procedimientos pudieron completarse exitosamente, aunque un paciente presentó fibrilación ventricular posprocedimiento y otro falleció durante el posoperatorio inmediato debido a trastorno grave de la coagulación. El seguimiento de los 12 pacientes restantes fue en promedio de 19 meses y la evolución fue favorable.

Conclusiones: Utilizando una estrategia híbrida, los resultados iniciales en términos de sobrevida y mejoramiento de parámetros hemodinámicos fueron adecuados. La selección de casos, el trabajo en equipo y el seguimiento apropiado son cruciales para lograr resultados satisfactorios.
\end{abstract}

Palabras clave: Procedimientos quirúrgicos cardíacos/métodos - Cardiopatías congénitas/cirugía

\section{ABSTRACT}

Background: The hybrid approach implies the close collaboration between surgical and interventional cardiology teams for the treatment of complex congenital heart diseases to reduce the number of interventions by using less invasive techniques, avoid the limitations due to the size of vascular accesses and the use of cardiopulmonary bypass.

Objectives: The aim of this study is to evaluate the indications, techniques, initial results and short-term follow-up of the hybrid approach.

Methods: We conducted a retrospective analysis of patients treated with hybrid approach between March 2014 and September 2020. Fourteen procedures were performed in 13 patients. Mean age was 16.8 years ( 1 day-13 years); $50 \%$ were neonates, $57 \%$ were male and mean weight was $9 \mathrm{~kg}(1.9-70 \mathrm{~kg})$. The hybrid procedures performed included stenting in coarctation of the aorta (4 patients), ductal stenting (3 patients), stenting of pulmonary artery branches (1 patient), neonatal aortic valvuloplasty (4 patients) and perventricular closure of muscular ventricular septal defect ( 2 patients). The access used was dissection of the carotid artery in 8 procedures and sternotomy in the remaining 6 .

Results: All the procedures were successfully completed, except for two patients: one died due to ventricular fibrillation after the procedure, and another in the immediate postoperative period due to severe coagulation abnormality. Mean follow-up of the remaining 12 patients was 19 months with favorable outcome.

Conclusions: The initial results with the use of a hybrid strategy were satisfactory in terms of survival and improvement of the hemodynamic parameters. The selection of cases, teamwork and appropriate follow-up are key factors to achieve satisfactory results.

Key words: Cardiac Surgical Procedures / methods - Heart Defects, Congenital / surgery

Rev Argent Cardiol 2021;89:124-129. http://dx.doi.org/10.7775/rac.es.v89.i2.19990

Recibido: 09/12/2020 - Aceptado: 04/02/2021

Dirección para separatas: Dr. Alejandro Peirone - Hospital Privado Universitario de Córdoba - Naciones Unidas 346 (5016) Córdoba, Argentina - E-mail: alepeirone@yahoo.com

Este trabajo obtuvo el Premio Kreutzer 2020 al mejor trabajo en cardiología pediátrica

\footnotetext{
${ }^{1}$ Hospital Privado Universitario de Córdoba.

${ }^{2}$ Hospital de Niños de la Santísima Trinidad de Córdoba.

${ }^{3}$ Clínica Privada del Sol de Córdoba.

${ }^{4}$ Centro Médico Especializado Diagnus de Córdoba.

${ }^{5}$ Instituto Universitario de Ciencias Biomédicas de Córdoba.
} 


\begin{tabular}{|c|c|c|c|}
\hline CCC & Cardiopatías congénitas complejas & SCIH & Síndrome de corazón izquierdo hipoplásico \\
\hline CEC & Circulación extracorpórea & CIV & Comunicación interventricular \\
\hline CoAo & Coartación de aorta & DAP & Ductus arterioso permeable \\
\hline ReCoAo & Recoartación de aorta & & \\
\hline
\end{tabular}

\section{INTRODUCCIÓN}

Durante la última década, hemos evidenciado una mejoría significativa en los resultados de cirugía por cardiopatías congénitas complejas (CCC) y también la expansión de las técnicas de hemodinamia intervencionista para el mismo fin. En el abordaje híbrido se muestra la colaboración estrecha de los equipos quirúrgicos y de hemodinamia, donde cada uno utiliza las mejores técnicas de su campo con el objetivo de disminuir el número de intervenciones a través de técnicas menos invasivas, de evitar las limitaciones dadas por el tamaño pequeño de los accesos vasculares en neonatos y lactantes y de reducir el tiempo de circulación extracorpórea (CEC) y parada circulatoria. El abordaje híbrido también permite la planificación en tiempo y forma de los casos más desafiantes que se beneficiarían con un procedimiento combinado y brinda opciones de tratamiento que se adaptan a las necesidades individuales. (1-4)

El objetivo de nuestro trabajo fue evaluar las indicaciones, las técnicas, los resultados iniciales y el seguimiento a corto plazo del abordaje híbrido en diferentes centros dedicados al tratamiento de pacientes con CCC.

\section{MATERIAL Y MÉTODOS}

Se efectuó un estudio multicéntrico descriptivo, observacional. Se incluyeron retrospectivamente los pacientes que fueron abordados de manera híbrida desde marzo de 2014 hasta mayo de 2020 en diferentes instituciones médicas de la ciudad de Córdoba. Todos los procedimientos fueron realizados por el mismo equipo médico. La indicación de abordaje híbrido en cada paciente fue acordada por los equipos de cardiología, de cirugía cardiovascular y de hemodinamia de cada centro. Se incluyeron en el análisis las características demográficas, el tipo de cardiopatía congénita, las vías de abordaje, el procedimiento híbrido realizado, sus complicaciones, los resultados iniciales y el seguimiento a corto plazo.

\section{Análisis estadístico}

Las variables categóricas se expresaron como porcentajes y las variables continuas como promedio e intervalo. Para el análisis estadístico se utilizó el paquete estadístico InfoStat/P (Universidad Nacional de Córdoba, 2018).

\section{Consideraciones éticas}

El estudio se realizó siguiendo las recomendaciones de la declaración de Helsinki para estudios observacionales. Fue aprobado por el Comité de Ética de los hospitales intervinientes, pero no incluyó firma de consentimiento informado por parte de los pacientes.

\section{RESULTADOS}

Desde marzo de 2014 hasta septiembre de 2020 se realizaron 14 procedimientos híbridos en 13 pacientes (Tabla 1). La edad media fue de 16,8 meses (intervalo: 1 día-13 años) y el 50\% de los pacientes eran neonatos. El peso medio fue de $9 \mathrm{~kg}$ (intervalo: $1,9-70 \mathrm{~kg}$ ) y el $57 \%$ eran de sexo masculino (Tabla 2). Los procedimientos realizados incluyeron implantación híbrida de stent en coartación de aorta (CoAo) (28\%), colocación de stent ductal (20\%), implante de stent en ramas pulmonares (7\%), valvuloplastia aórtica neonatal $(28 \%)$ y cierre perventricular de comunicación interventricular muscular (14\%) (Tabla 3).

Se realizaron 4 implantes de stent en CoAo, 2 en pacientes con diagnóstico de CoAo nativa de segmento largo y los otros 2 en pacientes con recoartación de aorta (ReCoAo), uno de ellos posquirúrgico y con marcado compromiso hemodinámico y el restante con una variante de síndrome de corazón izquierdo hipoplásico (SCIH) pos estadio de Glenn bidireccional, con estrechez distal de cirugía de Norwood de grado severo.

En un paciente con tetralogía de Fallot pos reparación completa que desarrolló estenosis grave de tubo colocado entre el ventrículo derecho y las ramas de las arterias pulmonares, asociada a estenosis difusa de grado severo de rama pulmonar izquierda, se realizó implante de stent intraoperatorio en dicha rama.

Se implantaron tres stents en ductus arterioso en circulación sistémica dependiente de aquel. Uno se implantó en un paciente con diagnóstico de estenosis valvular aórtica crítica fetal y disfunción/dilatación grave ventricular izquierda, a quien se le realizó dilatación valvular aórtica híbrida neonatal. En otro caso, se realizó durante el mismo procedimiento implante de stent ductal y cerclaje bilateral de ramas de arterias pulmonares en un paciente con hipoplasia significativa valvular y de cayado aórtico, asociada a CIV tipo mal alineamiento de tamaño grande. El tercer caso fue realizado en un paciente con diagnóstico de SCIH con cerclaje bilateral de ramas pulmonares y septostomía auricular previos.

Se realizaron dos cierres de CIV perventriculares, ambos defectos de tipo medio muscular, un procedimiento se asoció con extracción de cerclaje de arteria pulmonar y el otro con ligadura de ductus arterioso permeable (DAP).

Finalmente, se realizaron cuatro valvuloplastias aórticas con acceso híbrido en neonatos con diagnóstico de estenosis valvular de grado severo. 


\begin{tabular}{|c|c|c|c|c|c|c|c|c|}
\hline $\begin{array}{l}\text { Inter- } \\
\text { vención }\end{array}$ & Edad & $\begin{array}{l}\text { Peso } \\
(\mathrm{kg})\end{array}$ & Diagnóstico & Procedimiento & $\begin{array}{l}\text { Abordaje } \\
\text { anomalías } \\
\text { cardiacas }\end{array}$ & $\begin{array}{c}\text { Otras } \\
\text { simultáneos } \\
\text { adicionales }\end{array}$ & Procedimientos & Complicaciones \\
\hline 1 & $15 d$ & 2,5 & $\begin{array}{c}\text { ReCoAo crítica } \\
\text { arco transverso } \\
\text { distal }\end{array}$ & $\begin{array}{c}\text { Implante } \\
\text { stent aórtico }\end{array}$ & $\begin{array}{c}\text { Disección } \\
\text { CD }\end{array}$ & & & \\
\hline 2 & $7 \mathrm{~m}$ & 5,2 & $\begin{array}{c}\text { CoAo } \\
\text { yuxtaductal } \\
\text { crítica + } \\
\text { hipoplasia } \\
\text { istmo }\end{array}$ & $\begin{array}{l}\text { Implante stent } \\
\text { aórtico }\end{array}$ & $\begin{array}{c}\text { Disección } \\
\text { CD }\end{array}$ & & & $\begin{array}{c}\text { FV, torsión de } \\
\text { punta }\end{array}$ \\
\hline 3 & $12 \mathrm{~m}$ & 11 & $\begin{array}{c}\text { CoAo } \\
\text { yuxtaductal } \\
\text { crítica }+ \\
\text { hipoplasia } \\
\text { istmo }\end{array}$ & $\begin{array}{l}\text { Implante stent } \\
\text { aórtico }\end{array}$ & $\begin{array}{c}\text { Disección } \\
\text { CD }\end{array}$ & & & \\
\hline 4 & $22 d$ & 8 & ReCoAo crítica & $\begin{array}{l}\text { Implante stent } \\
\text { aórtico }\end{array}$ & $\begin{array}{l}\text { Disección } \\
\text { CD } \\
\text { Cirugía } \\
\text { Norwood, } \\
\text { Glenn }\end{array}$ & $\begin{array}{c}\text { Variante } \\
\text { SCIH- }\end{array}$ & & \\
\hline 5 & $13 a$ & 70 & $\begin{array}{c}\text { Estenosis RPI } \\
\text { crítica }\end{array}$ & $\begin{array}{c}\text { Implante stent } \\
\text { RPI } \\
\text { (CEC) }\end{array}$ & $\begin{array}{c}\text { Re- } \\
\text { esternotomía }\end{array}$ & $\begin{array}{c}\text { TOF } \\
\text { corregida }\end{array}$ & & $\begin{array}{l}\text { Sangrado masivo } \\
\text { Falleció }\end{array}$ \\
\hline 6 & $27 d$ & 2,5 & $\begin{array}{l}\text { Hipoplasia } \\
\text { VAo y AAo } \\
\text { severa, CIV, } \\
\text { DAP }\end{array}$ & $\begin{array}{c}\text { Implante stent } \\
\text { ductal }\end{array}$ & Esternotomía & CIA OS & $\begin{array}{c}\text { Cerclaje ramas } \\
\text { de AP }\end{array}$ & \\
\hline 7 & $17 d$ & 2,3 & $\begin{array}{c}\text { Estenosis VAo } \\
\text { crítica pos } \\
\text { valvuloplastia } \\
\text { híbrida }\end{array}$ & $\begin{array}{c}\text { implante stent } \\
\text { ductal }\end{array}$ & $\begin{array}{c}\text { Reapertura } \\
\text { esternotomía } \\
\text { DAP } \\
\text { restrictivo }\end{array}$ & $\begin{array}{c}\text { Cerclaje } \\
\text { ramas de AP, }\end{array}$ & & \\
\hline 8 & $2 m$ & 1,9 & $\mathrm{SClH}$ & $\begin{array}{c}\text { Implante stent } \\
\text { ductal }\end{array}$ & $\begin{array}{c}\text { Reapertura } \\
\text { esternotomía } \\
\text { septostomía } \\
\text { auricular }\end{array}$ & $\begin{array}{c}\text { Cerclaje } \\
\text { ramas AP, }\end{array}$ & & \\
\hline 9 & $1 \mathrm{~d}$ & 2,5 & $\begin{array}{c}\text { Estenosis VAo } \\
\text { crítica }\end{array}$ & $\begin{array}{c}\text { Valvuloplastia } \\
\text { aórtica }\end{array}$ & $\begin{array}{c}\text { Disección } \\
\text { CD }\end{array}$ & & & \\
\hline 10 & $1 \mathrm{~m}$ & 2,7 & $\begin{array}{c}\text { Estenosis VAo } \\
\text { crítica }\end{array}$ & $\begin{array}{c}\text { Valvuloplastia } \\
\text { aórtica }\end{array}$ & $\begin{array}{c}\text { Disección } \\
\text { CD }\end{array}$ & & & \\
\hline 11 & $2 \mathrm{~m}$ & 4 & $\begin{array}{l}\text { Estenosis } \\
\text { VAo crítica }\end{array}$ & $\begin{array}{c}\text { Valvuloplastia } \\
\text { aórtica }\end{array}$ & $\begin{array}{c}\text { Disección } \\
\text { CD }\end{array}$ & & & $\begin{array}{l}\text { I VAo leve a } \\
\text { moderada }\end{array}$ \\
\hline 12 & $22 d$ & 3 & $\begin{array}{l}\text { Estenosis } \\
\text { VAo crítica }\end{array}$ & $\begin{array}{c}\text { Valvuloplastia } \\
\text { aórtica }\end{array}$ & $\begin{array}{c}\text { Disección } \\
\text { CD }\end{array}$ & $\begin{array}{l}\text { Síndrome } \\
\text { Shone }\end{array}$ & & \\
\hline 13 & $7 \mathrm{~m}$ & 4,5 & $\begin{array}{l}\text { CIV muscular } \\
\text { apical }\end{array}$ & $\begin{array}{c}\text { Cierre } \\
\text { perventricular } \\
\text { CIV }\end{array}$ & $\begin{array}{c}\text { Esternotomía } \\
\text { troncoAP }\end{array}$ & $\begin{array}{l}\text { CoAo } \\
\text { reparada, } \\
\text { cerclaje }\end{array}$ & $\begin{array}{l}\text { Extracción } \\
\text { cerclaje AP }\end{array}$ & \\
\hline 14 & $22 \mathrm{~m}$ & 8 & $\begin{array}{l}\text { CIV medio } \\
\text { muscular }\end{array}$ & $\begin{array}{c}\text { Cierre } \\
\text { perventricular } \\
\text { CIV }\end{array}$ & Esternotomía & DAP & Ligadura DAP & \\
\hline
\end{tabular}

AAo, arco aórtico; AP, arteria pulmonar; $C D$, carótida derecha; $C E C$, circulación extracorpórea; CIA OS, comunicación interauricular ostium secundum; CIV, comunicación interventricular; CoAo, coartación aorta; DAP, ductus arterioso permeable; FV, fibrilación ventricular; I VAo, insuficiencia válvula aórtica; Re CoAo, recoartación de aorta; RPI, rama pulmonar izquierda; SCIH, síndrome de corazón izquierdo hipoplásico; TOF, tetralogía de Fallot; VAo, válvula aórtica; 


\begin{tabular}{ll}
\hline Tabla 2. Características demográficas y abordajes \\
\hline \\
Edad & 16,8 meses (1 día-13 años) \\
\hline Peso & $9 \mathrm{~kg}(1,9-70 \mathrm{~kg})$ \\
\hline Sexo & $57 \%$ masculino/ 43\% femenino \\
\hline Abordaje & Disección carótida derecha $57 \%$ \\
& Esternotomía 43\%
\end{tabular}

Tabla 3. Procedimientos híbridos realizados

\begin{tabular}{|lc|}
\hline Implante stent aórtico & $4(28,5 \%)$ \\
\hline Implante stent ductal & $3(21,4 \%)$ \\
\hline Implante stent rama pulmonar & $1(7,1 \%)$ \\
\hline Valvuloplastia aórtica & $4(28,5 \%)$ \\
\hline Cierre comunicación interventricular & $2(14,2 \%)$ \\
\hline
\end{tabular}

El abordaje híbrido se llevó a cabo mediante disección de arteria carótida derecha en 8 pacientes, 4 de ellos para realización de valvuloplastia aórtica y los restantes para colocación de stent en CoAo. En 6 pacientes el acceso fue mediante esternotomía mediana (dos cierres de CIV perventriculares, dos colocaciones de stents ductales mediante punción de tracto de salida de ventrículo derecho, una colocación de stent ductal mediante punción de tronco de arteria pulmonar y una colocación de stent en rama pulmonar izquierda mediante apertura quirúrgica del tronco de la arteria pulmonar). Dos de las intervenciones fueron realizadas en pacientes que requirieron reapertura de esternotomía previa y en un paciente se realizó reapertura de esternotomía por cuarta vez, dicho procedimiento fue realizado con CEC y parada circulatoria. No se describieron complicaciones relacionadas con el abordaje.

Todos los procedimientos programados lograron completarse exitosamente. Entre las complicaciones observadas se describe un paciente con colocación de stent en CoAo que desarrolló fibrilación ventricular a las 3 horas del procedimiento y sangrado en arteria ilíaca interna derecha que requirió reparación quirúrgica a los 7 días del procedimiento inicial. En otro caso, un paciente de 13 años falleció por sangrado incontrolable pos cuarta reesternotomía por recambio de tubo entre ventrículo derecho y tronco de arteria pulmonar e implante híbrido de stent en rama pulmonar izquierda, procedimiento complejo realizado con CEC y parada circulatoria.

Luego del alta hospitalaria, todos los pacientes fueron controlados clínicamente y mediante ecocardiografía Doppler color con un promedio de seguimiento de 19 meses (1-75 meses) y tuvieron una evolución favorable. Los 4 pacientes que requirieron stent por obstrucción del arco aórtico no presentaron gradiente significativo. Los 2 pacientes que requirieron dispositivos para cierre de CIV evolucionaron sin cortocircuitos residuales. De los 4 pacientes con estenosis valvular aórtica neonatal, 3 permanecen sin necesidad de reintervención. En uno de ellos, debido al incremento de gradiente significativo, fue necesaria una segunda valvuloplastia aórtica percutánea, que tuvo buen resultado. De los 3 pacientes con implantación de stent ductal, 2 se encuentran en planes de cirugía definitiva de su CCC. Al restante se le realizó extracción de cerclaje percutáneo bilateral de ramas pulmonares mediante dilatación con balón y oclusión percutánea del stent ductal mediante implante de tapón vascular, con buen resultado.

\section{DISCUSIÓN}

Históricamente, los cirujanos cardiovasculares y los cardiólogos intervencionistas han mantenido una relación competitiva. Con el avance de las técnicas hemodinámicas, coincidente con la mejoría observada en los resultados quirúrgicos en el manejo de las CCC, es lógico pensar que los nuevos desafíos para ambas disciplinas podrán ser enfrentados y resueltos más eficazmente mediante un esfuerzo colaborativo. Para alcanzar este objetivo, es claro que debe haber ausencia de competencia profesional o económica y respeto mutuo por los talentos y las limitaciones individuales. Además, se deben mantener discusiones francas entre los miembros del equipo multidisciplinario cardiovascular interviniente.

En nuestro Programa de Atención de Pacientes con Cardiopatías Congénitas del Niño y del Adulto, comenzamos con los procedimientos híbridos con objetivos definidos, que incluyeron la reducción de la morbimortalidad de pacientes complejos, la mejoría de la calidad de vida, el suministrar un cuidado individual más eficiente y costo/efectivo y, sobre todo, el reducir el impacto acumulativo de múltiples intervenciones a los que estos pacientes complejos se enfrentan en su vida. Entre las ventajas que esta técnica híbrida colaborativa ofrece, encontramos la rapidez con la que los procedimientos percutáneos son realizados, ya que cuentan con acceso más directo y seguro, lo que permite una adecuada inspección anatómica y la actuación temprana del cirujano en caso de ser necesario. También se destaca la posibilidad de realizar procedimientos con escasas limitaciones en cuanto a edad y/o peso y que, en muchos casos, se evita el uso de CEC. (5)

En los casos que requieren colocación de stent intraoperatorio en ramas de arterias pulmonares, puede adicionarse la utilización de broncoscopio rígido o flexible para lograr una visualización directa "en vivo" de la lesión y efectuar el control posterior a la intervención. (6) Además, mediante la técnica híbrida se evita el compromiso de ramas lobares y segmentarias más próximas, disminuye el tiempo de CEC y se evita la necesidad de realizar disecciones extensas retro- $y$ 
paraaórticas, que son necesarias en muchos casos de reparación solo quirúrgica. (7)

En las intervenciones para cierre perventricular de CIV musculares, eliminamos el problema del acceso vascular en pacientes pequeños y pudimos realizar las intervenciones a edades más tempranas (pacientes menores de $5 \mathrm{~kg}$ ); también evitamos el uso de vainas de gran calibre para colocación del dispositivo, disminuimos o abolimos la utilización de CEC y, en casos de defectos del septum interventricular complejos que se extienden hacia el ápex ventricular, se disminuye la persistencia de cortocircuitos residuales. Alternativamente, también puede utilizarse en pacientes que presentan contraindicaciones para CEC o en aquellos con intento fallido de cierre percutáneo o quirúrgico. $(8,9)$

En cuanto al tratamiento de la estenosis aórtica neonatal, realizamos la valvuloplastia aórtica mediante acceso carotídeo, ya que, en neonatos, es mayor el diámetro de la arteria carótida comparado con el diámetro de la arteria femoral. Dicho acceso previene las complicaciones del acceso femoral, como es la trombosis en pacientes de alto riesgo, sobre todo, neonatos menores de $4 \mathrm{~kg}$. También facilita el avance del catéter en anatomías tortuosas o de ángulo agudo para realizar ciertos procedimientos y provee una vía de acceso más segura y efectiva hacia la lesión a tratar. $(10,11)$ También evita el daño potencial de la válvula mitral cuando la técnica percutánea seleccionada es el acceso anterógrado a la válvula aórtica. (12-14) La utilización del mismo abordaje híbrido carotídeo para la colocación de stents en CoAo y ReCoAo nos permite la colocación de vainas de mayor calibre, por las cuales se avanzan stents que pueden ser redilatables hasta diámetros compatibles con la vida adulta. $(15,16)$

El abordaje híbrido del SCIH es una alternativa de tratamiento establecida. Se presenta como un enfoque inicial o estadio I de tratamiento paliativo en pacientes con variantes de SCIH que incluye tres etapas (cerclaje bilateral de ramas pulmonares, stent en DAP y septostomía auricular). Es un abordaje útil en pacientes de alto riesgo, ya que evita la CEC y la hipotermia cardíaca en la etapa neonatal. Los pacientes de alto riesgo incluyen aquellos con bajo peso al nacer $(<2,5$ $\mathrm{kg}$ ), prematurez, disfunción ventricular, hipoplasia grave de aorta ascendente, malformaciones genéticas y otras comorbilidades graves. $(17,18)$ Los resultados del abordaje híbrido en SCIH son alentadores, lo que ratifica la importancia de la colaboración entre los equipos tratantes para afrontar el manejo de estos pacientes con cardiopatías congénitas complejas graves.

En nuestro programa, la estrategia híbrida para pacientes que presentan SCIH es llevada a cabo en la sala de hemodinamia, donde el equipo cardiovascular realiza la esternotomía y coloca inicialmente los cerclajes de ambas ramas de las arterias pulmonares. Seguidamente, se realiza punción del tracto de salida del ventrículo derecho (o, alternativamente, del tronco de arteria pulmonar), donde se coloca una vaina corta para realizar angiografía inicial. En esta última, se observa la indentación de ambos cerclajes de ramas pulmonares y la anatomía/diámetro del ductus arterioso. Finalmente, por esta vaina se avanza y se impacta el stent seleccionado en la estructura ductal. En una segunda intervención, si es necesario, se realiza la septostomía auricular percutánea.

Esta estrategia híbrida para el abordaje del SCIH se fundamenta en que evitando la realización de CEC y la parada circulatoria durante el período neonatal mejorará la función miocárdica a largo plazo. Además, demorando la reconstrucción del arco aórtico (que requiere CEC/parada circulatoria) hasta los 4-6 meses de vida, mejorarán los resultados neurológicos a largo plazo. Aunque es todavía una hipótesis, creemos que un lactante menor de 4-6 meses que sale de quirófano con "circulación en serie" (anastomosis de Glenn) es más estable que un neonato fuera de quirófano con "circulación balanceada" (anastomosis de Blalock-Taussig o Sano) luego de una intervención similar. El debate está planteado, por lo que, en la actualidad, se están llevando a cabo múltiples estudios de investigación para responder a estos interrogantes.

\section{CONCLUSIÓN}

En nuestra experiencia multicéntrica pionera, los resultados iniciales utilizando una estrategia híbrida para el tratamiento de CCC en términos de sobrevida y mejoramiento de parámetros hemodinámicos fueron adecuados. Los procedimientos son técnicamente seguros y eficaces. La planificación detallada y el trabajo en equipo son la clave para el éxito de la técnica híbrida. Se requerirá un mayor número de casos y un tiempo de seguimiento más alejado para confirmar las observaciones iniciales.

\section{Declaración de conflicto de intereses}

Los autores declaran que no poseen conflicto de intereses.

(Véase formulario de conflicto de intereses de los autores en la web / Material suplementario).

\section{BIBLIOGRAFÍA}

1. Galantowicz M. Hybrid Procedures: A Surgeon's Viewpoint on the Next 10 Years. Pediatr Cardiol 2020;41:514-21. https://doi. org/10.1007/s00246-020-02299-x

2. Gupta A, Amin Z. Popular Hybrid Congenital Heart Procedures without Cardiopulmonary Bypass. Front Surg 2017;4:9. https://doi. org/10.3389/fsurg.2017.00009

3. Russell MR, Blais B, Nia N, Levi D. The Potential Impact and Timeline of Engineering on Congenital Interventions. Pediatr Cardiol 2020;41:522-38. https://doi.org/10.1007/s00246-020-02335-w

4. Kenny D, Galantowicz M, Cheatham J, Hijazi Z. Hybrid Management Techniques in the Treatment of the Neonate with Congenital Heart Disease. In Hemodynamics and Cardiology: Neonatology Questions and Controversies. WB Saunders; 2012. p 521-35. https:// doi.org/10.1016/B978-1-4377-2763-0.00027-5

5. Parody-Cuerda G, Coserria-Sánchez F, Adsuar Gómez A, GarcíaHernández J, Manso-García B, Hosseinpour A. Pulmonary valve perforation and stent implantation in the right ventricle outflow tract using a hybrid procedure as an alternative to a central shunt. 
Rev Esp Cardiol (Engl Ed) 2018;71:1081-2. https://doi.org/10.1016/j. recesp.2017.09.027

6. Ebrahim M, Hagood J, Moore J, El $\square$ Said H. Bronchoscopic guidance of endovascular stenting limits airway compression. Catheter Cardiovasc Interv 2015;85:832-6. https://doi.org/10.1002/ccd.25772 7. Menon S, Cetta F, Dearani J, Burkhart H, Cabalka A, Hagler D. Hybrid intraoperative pulmonary artery stent placement for congenital heart disease. Am J Cardiol 2008;102:1737-41. https://doi. org/10.1016/j.amjcard.2008.07.061

8. Michel $\square$ Behnke I, Ewert P., Koch A, Bertram H, Emmel M, Fischer $\mathrm{G}$, et al. Device closure of ventricular septal defects by hybrid procedures: a multicenter retrospective study. Catheter Cardiovasc Interv 2011;77:242-51. https://doi.org/10.1002/ccd.22666

9. Molaei A, Afrasiabi A, Bilejani E, Samadi M. Perventricular muscular ventricular septal defect (VSD) closure under epicardial echocardiography guidance: A case report. J Tehran Univ Heart Cent 2017;12:138-41.

10. Justino H, Petit C. Percutaneous common carotid artery access for pediatric interventional cardiac catheterization. Circ Cardiovasc Interv 2012;9:e003003. https://doi.org/10.1161/CIRCINTERVENTIONS.115.003003

11. Borghi A, Agnoletti G, Poggiani C. Surgical cutdown of the right carotid artery for aortic balloon valvuloplasty in infancy: midterm follow-up. Pediatr Cardiol 2001;22:194-7. https://doi.org/10.1007/ s002460010202

12. Weber H, Mart C, Kupferschmid J, Myers J, Cyran S. Transcarotid balloon valvuloplasty with continuous transesophageal echocardiographic guidance for neonatal critical aortic valve stenosis: an alternative to surgical palliation. Pediatr Cardiol 1998; 19:212-7. https:// doi.org/10.1007/s002469900287

13. Robinson B, Brzezinska-Rajszys G, Weber H, Ksiazyk J, Fricker F, Fischer D, et al. Balloon aortic valvotomy through a carotid cutdown in infants with severe aortic stenosis: results of the multi-centric registry. Cardiol Young 2000;10:225-32. https://doi.org/10.1017/ S104795110000915X

14. Maeno Y, Akagi T, Hashino K, Ishii M, SugimuraT, Takagi J, et al. Carotid artery approach to balloon aortic valvuloplasty in infants with critical aortic valve stenosis. Pediatr Cardiol 1997;18:288-91. https://doi.org/10.1007/s002469900176

15. Suradi H, Hijazi Z. Current management of coarctation of the aorta. Glob Cardiol Sci Pract 2015;2015:44. https://doi.org/10.5339/ gesp. 2015.44

16. McLennan D, Caputo M, Taliotis D. severe aortic stenosis and severe Coarctation of the aorta: a Hybrid approach to treatment. Front Surg 2017;4:16. https://doi.org/10.3389/fsurg.2017.00016

17. Bacha E, Hijazi Z. Hybrid procedures in pediatric cardiac surgery. En Seminars in thoracic and cardiovascular surgery: Pediatric Cardiac Surgery Annual. WB Saunders 2005; 8 p.78-85. https://doi. org/10.1053/j.pcsu.2005.01.001

18. Agrawal H, Alkashkarib W, Kenny D. Evolution of hybrid interventions for congenital heart disease. Expert Rev of Cardiovasc Ther 2017;15:257-66. https://doi.org/10.1080/14779072.2017.1307733 